\title{
Clinico-pathology and hemato-biochemistry responses in buffaloes infected with Pasteurella multocida type B:2 immunogen outer membrane protein
}

\begin{abstract}
The aim of this study was to investigate the clinico-pathology and haemato-biochemistry alterations in buffaloes inoculated with Pasteurella multocida type B:2 immunogen outer membrane protein via subcutaneous and oral routes. Nine buffalo heifers were divided equally into 3 treatment groups. Group 1 was inoculated orally with $10 \mathrm{~mL}$ of phosphate buffer saline (PBS); Group 2 and 3 were inoculated with $10 \mathrm{~mL}$ of outer membrane protein broth subcutaneously and orally respectively. Group 2 buffaloes showed typical haemorrhagic septicaemia clinical signs and were only able to survive for $72 \mathrm{~h}$ of the experiment. However, Group 3 buffaloes were able to survive throughout the stipulated time of 21 days of experiment. There were significant differences $(p<0.05)$ in the rectal temperature between the experimental and control group. In the hematology and biochemistry findings, there were significant differences $(\mathrm{p}<0.05)$ in packed cell volume, mean corpuscular volume, mean corpuscular haemoglobin concentration, leukocytes, band neutrophils, segmented neutrophils, lymphocytes, eosinophils, basophils, gamma glutamyl transferase, total protein, and globulin between Group 2 and control group. In contrast, Group 3 and control group revealed significant differences $(\mathrm{p}<0.05)$ in erythrocytes, haemoglobin, mean corpuscular haemoglobin concentration, segmented neutrophils, lymphocytes, monocytes, eosinophils, basophils, thrombocytes, gamma glutamyl transferase, total protein, globulin, and albumin:globulin ratio. In Group 2 buffaloes, there were gross lesions observed in the lung, trachea, heart, liver, spleen, kidney and submandibulae lymph nodes. In contrast, lesions were only observed in the lung, and liver of Group 3 buffaloes. There were significant differences $(\mathrm{p}<0.05)$ in hemorrhage and congestion; necrosis and degeneration; and inflammatory cells infiltration between experimental groups and control group. However, there were no significant differences $(p>0.05)$ in edema between groups except for the lung. This study was a proof that oral route infection of Pasteurella multocida type B:2 immunogen outer membrane protein can be used to stimulate host cell.
\end{abstract}

Keyword: Buffalo heifers; Clinico-pathology; Haemato-biochemistry responses; Oral inoculation; Outer membrane protein; Pasteurella multocida type B:2; Subcutaneous inoculation 